\title{
An OTDR and Gratings assisted Multifunctional Fiber Sensing System
}

\author{
Xiangchuan Wang, Zhijun Yan, Feng Wang, Chengbo Mou, Zhongyuan Sun, Xuping Zhang and Lin \\ Zhang
}

\begin{abstract}
We report a distributed multifunction fiber sensing network based on weak-fiber Bragg gratings (WFBGs) and long period fiber grating (LPG) assisted OTDR system. The WFBGs are applied for temperature, strain and vibration monitoring at key-positions, and the LPG is used as a linear filter in the system to convert the wavelength shift of WFBGs caused by environmental change into the power change. The simulation results show that it is possible to integrate more than 4472 WFBGs in the system when the reflectivity of WFBGs is less than $10^{-5}$. Besides, the reflected light of weak FBGs and the back-Rayleigh scattering along the whole fiber can also be detected which makes distributed bend sensing possible. As an experimental demonstration, we have used 3 WFBGs UV-inscribed with $50 \mathrm{~m}$ interval at the end of a $2.6 \mathrm{~km}$ long fiber, which part was subjected for temperature, strain and vibration sensing, respectively. The results show strain and temperature sensitivities are $4.2 \times 10^{-4} / \mu \varepsilon$ and $5.9 \times 10^{-3} /{ }^{\circ} \mathrm{C}$, respectively. Detection of multiple vibrations and single vibration with the broad frequency band up to $500 \mathrm{~Hz}$ are also achieved. In addition, distributed bend sensing which could be simultaneously realized in this system has been proposed.
\end{abstract}

Index Terms-Optical fiber measurements; Optical time domain reflectometry; Vibration measurement; optical fiber gratings.

\section{INTRODUCTION}

$\mathrm{O}$ PTICAL time domain reflectometry (OTDR) and fiber Bragg gratings (FBGs) are the most useful fiber sensors in modern optical sensing applications [1,2]. OTDR can be used for long-distance distributed sensing, which has great potential

This work was supported in part by the National Basic Research Program of China (973 Program) under Grant No. 2010CB327803, National Natural Science Foundation of China under Grant No. 61107074 and 61027017 and the China Scholar Council (CSC).

Z. Yan, C. Mou, Z. Sun and L. Zhang are now with Aston Institute of Photonic Technologies, Aston University, Birmingham, B4 7PG, UK (email: yanz1@aston.ac.uk; c.mou1@aston.ac.uk; sunz5@aston.ac.uk; 1.zhang@aston.ac.uk).

F. Wang is now with the Institute of Optical Communication Engineering, Nanjing University, Nanjing, 210093, China (email: hellowangfeng@gmail.com; xpzhang@nju.edu.cn).

$\mathrm{X}$. Zhang (Corresponding author) is now with Institute of Optical Communication Engineering, Nanjing University, Nanjing, 210093, China and Key Laboratory of Modern Acoustics, Nanjing University, Nanjing, 210093, China (email: xpzhang@nju.edu.cn)

$\mathrm{X}$. Wang is now with the Institute of Optical Communication Engineering, Nanjing University, Nanjing, 210093, China, Key Laboratory of Modern Acoustics, Nanjing University, Nanjing, 210093, China and Aston Institute of Photonic Technologies, Aston University, Birmingham, B4 7PG, UK (email: nadazdh@163.com) in perimeter security, structure health and communication channel loss monitoring etc. [3,4]. However, OTDR does not offer high accuracy measurement for some sensing applications, such as strain, temperature and vibration detection. Over the last fifteen years, FBGs have been widely used for strain and temperature monitoring [5,6]. By integrating FBGs along the single mode fiber (SMF), quasi-distributed fiber sensing can be achieved $[7,8]$. However, the total number of FBGs is limited in these systems. Using weak FBGs, the number of integrated FBGs can be greatly improved, which promotes the development of FBG-based quasi-distributed fiber sensing capability [9-11]. On the other hand, the signal demodulation of FBG-based sensing systems using spectral analysis is relatively complex and high cost, which limits their applications. For example, it is hard to detect vibration, especially multiple vibrations, along the sensing system using the FBG based sensors due to long demodulation time. Long period fiber gratings (LPGs) have been used as linear filters for converting the spectral demodulation to simple power detection,, which greatly increases the response speed of the demodulation system, as demonstrated in vibration detection reported in ref. [12]. However, detecting multiple vibrations using such a method still needs to be further studied.

In practice, especially for large-scale structure monitoring, distributed sensors are necessary and it is also important to monitor temperature, strain and vibration simultaneously at key-positions. In this paper, an enhanced fiber sensing system based on LPG and weak FBG (WFBG) array assisted OTDR for multiple parameters distributed sensing is proposed. WFBGs as sensing elements at the key positions can be fabricated along the fiber, whose entire sensing spectral range can match with the linear filtering region of an LPG. Fallon et al have reported such a scheme [13], in which the attenuation is linearly changing as the changing of FBG wavelength, so the LPG acted as a wavelength to power converter to transfer the wavelength information of FBGs into power variations. Using the OTDR technology, the transferred power information of each WFBG can be detected for temperature, strain and vibration sensing at key-position while the WFBG's position is located by time-domain analysis. As WFBGs are part of the long fiber OTDR system, the power of Rayleigh scattering along the whole sensing fiber can also be obtained for distributed sensing, such as the detections for bending, loading, breakpoints and so on. The combination of WFBG and OTDR techniques would make it possible to implement a system 
capable of multi-function, distributed and key-position sensing.

\section{II.PRINCIPLE OF MEASUREMENT}

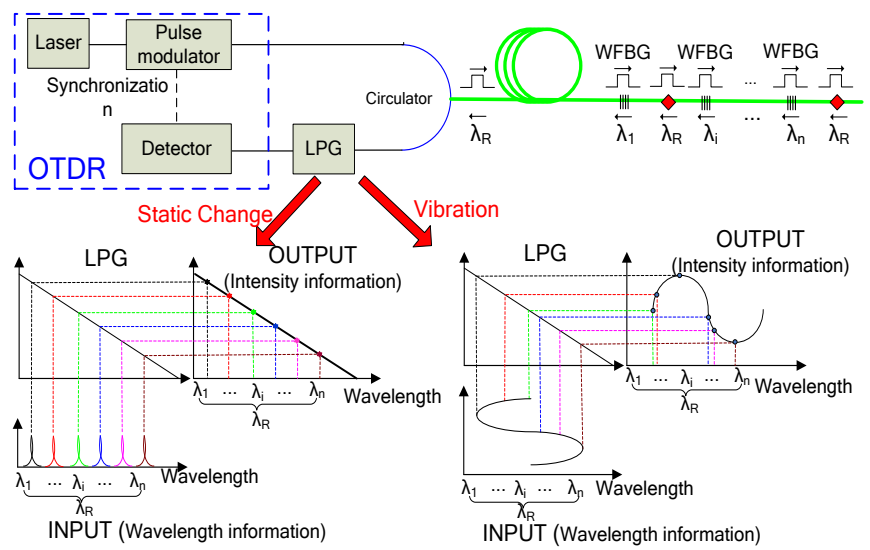

Fig.1: The principle of proposed distributed and key-position fiber sensing system.

Fig.1 shows the configuration of proposed distributed and key-position fiber sensing system. In the figure, a pulsed broadband light from OTDR output is launched into the sensing fiber integrating WFBGs in the end part of the fiber. When the pulse arrives at the WFBGs position, the light will be reflected by WFBGs. The reflected light is first filtered by an LPG, then received by OTDR. Due to the function of LPG, the power of the light detected by OTDR will vary linearly with the wavelength of reflected light. The linear relationship between wavelength and the power transfer function of the LPG can be written as:

$$
\mathrm{P}_{\text {OUT }}=\left(\mathrm{C}_{1} \lambda_{\mathrm{i}}+\mathrm{C}_{2}\right) \mathrm{P}_{\text {In }}
$$

Where $\lambda_{i}$ is the wavelength of light injected into the LPG; $P_{\text {OUT }}$ is the output power of the LPG; $\mathrm{C}_{1}$ and $\mathrm{C}_{2}$ are conditional parameters depending on the spectrum of the $\mathrm{LPG}$ and $\mathrm{P}_{\mathrm{In}}$ is the power of the input light.

The wavelength shift of WFBG caused by environmental change such as strain or temperature [1] can be expressed as:

$$
\Delta \lambda=\left(1-\mathrm{p}_{\mathrm{e}}\right) \varepsilon+\left(\alpha_{\Lambda}+\alpha_{\mathrm{n}}\right) \Delta \mathrm{T}
$$

Where $\Delta \lambda$ is the wavelength shift; $\varepsilon$ and $\Delta \mathrm{T}$ are the strain and temperature change, respectively; $p_{e}$ is the effective strain-optic constant; $\alpha_{\Lambda}$ and $\alpha_{\mathrm{n}}$ are the thermal expansion coefficient and thermo-optical coefficient of fiber respectively.

By substituting Eq.2 into Eq.1, the wavelength shift of WFBG can be converted into the power change by the LPG, which is described as:

$$
\Delta \mathrm{P}=\mathrm{P}_{\mathrm{In}} \cdot\left[\mathrm{C}_{1}\left(1-\mathrm{p}_{\mathrm{e}}\right) \varepsilon+\mathrm{C}_{1}\left(\alpha_{\Lambda}+\alpha_{\mathrm{n}}\right) \Delta \mathrm{T}\right]
$$

From Eq.3, we can find the power of the reflected light detected by OTDR will linearly change with strain or temperature. Therefore the changing of WFBGs caused by strain or temperature can be interrogated by just detecting the power change of the reflected light, instead of demodulating the wavelength shift. To avoid the fluctuation of the power of input light, the parameter $\mathrm{Q}$ is used to calibrate temperature and strain sensing:

$$
\mathrm{Q}=\Delta \mathrm{P} / \mathrm{P}_{\mathrm{In}}=\mathrm{C}_{1}\left(1-\mathrm{p}_{\mathrm{e}}\right) \varepsilon+\mathrm{C}_{1}\left(\alpha_{\Lambda}+\alpha_{\mathrm{n}}\right) \Delta \mathrm{T}
$$

When WFBGs are subjected to vibrations, the wavelengths will shift dynamically. As shown in Fig.1 and Eq.5, the power will change correspondingly with the vibration.

$$
\Delta \mathrm{P}(\mathrm{t}) / \mathrm{P}_{\text {In }}=\mathrm{C}_{1} \cdot \mathrm{K}_{\mathrm{f}} \cdot \mathrm{A} \cdot \cos (\omega \mathrm{t}+\varphi)
$$

Where $K_{f}$ is the transfer coefficient depending on the parameters of fiber; $\mathrm{A}$ is the amplitude of vibration while $\omega$ and $\varphi$ are the frequency and phase of the vibration. Thus it is possible to detect the vibration by transferring the power change into frequency domain by FFT.

OTDR technology is used to locate and distinguish vibrations along the entire fiber. As the reflected light of WFBGs would go backward at different time when the pulse travels along the sensing fiber using OTDR technology, vibrations around different WFBGs can be located and distinguished via time. Using the above mentioned method to obtain the filtered power of light from each WFBG, only one pulse is required in the sensing fiber each time. Therefore, the period of pulse should be at least:

$$
\Delta \mathrm{T}=\frac{2 \cdot \mathrm{L}}{\mathrm{c} / \mathrm{n}}
$$

Where $\mathrm{L}$ is the length of the sensing fiber, $\mathrm{c}$ is the speed of the light and $\mathrm{n}$ is the refraction index. Because there is only one effective sample point for each pulse along the whole sensing fiber, the actual sampling rate for vibration is:

$$
\mathrm{f}_{\mathrm{s}}=\frac{1}{\Delta \mathrm{T}}=\frac{\mathrm{c} / \mathrm{n}}{2 \cdot \mathrm{L}}
$$

According to the Nyquist theorem, the highest frequency of the vibration that can be detected is:

$$
\mathrm{f}=\mathrm{f}_{\mathrm{s}} / 2=\frac{\mathrm{c} / \mathrm{n}}{4 \cdot \mathrm{L}}
$$

Because WFBGs at different locations can be distinguished by using OTDR detection, it is not necessary to fabricate WFBGs with different wavelengths. Therefore, the number of the integrated WFBGs will not be limited by the bandwidth of input light source and the spectral resolution of the system, which greatly improves the interrogation e capacity.

Besides achieving the quasi-distributed sensing function, distributed sensing can also be achieved in this system. When the pulse arrives at non-WFBG area, the generated Rayleigh scattering light will also pass through the LPG as shown in Fig.1. Because the reflected light at the non-WFBGs area is from Rayleigh scattering, for which the wavelength $\lambda_{R}$ is not affected by the environment, the LPG in the system only contributed a constant attenuation to Rayleigh scattering signal which is not involved in distributed sensing achieved by the OTDR system.

\section{THEORETICAL ANALYSIS OF THE SENSING NETWORK CAPACITY}

In the system, the pulse reflected by WFBGs and received by the OTDR system will experience different paths (See in Fig. 2). As it shown in Fig. 2, the pulse experienced single reflection from WFBG3 will have the same travel distance as the one experienced multi-reflections from WFBG2-WFBG1-WFBG2. But they will have different powers, which can be described as [10]:

$$
\mathrm{P}_{\mathrm{OUT} 1}=(1-\mathrm{r})^{2(\mathrm{i}-1)} \cdot \mathrm{r} \cdot 10^{\left(\frac{\alpha \mathrm{L}}{5}+1\right)} \cdot \mathrm{P}_{\mathrm{In}}
$$




$$
\mathrm{P}_{\text {OUT2 }}=\mathrm{K} \cdot(1-\mathrm{r})^{2(\mathrm{i}-2)} \cdot \mathrm{r}^{3} \cdot 10^{\frac{\alpha \mathrm{L}}{5}+1} \cdot \mathrm{P}_{\mathrm{In}}
$$

Where $r$ is the reflectivity of WFBG; $i$ is the ith WFBG; $\alpha$ is the attenuation of the fiber; $L$ is the length that the light experiences; $\mathrm{K}=\frac{(\mathrm{i}-1)(\mathrm{i}-2)}{2}$. The Rayleigh scattering power can be described as:

$$
\mathrm{P}_{\text {OUTR }}=(1-\mathrm{r})^{2(\mathrm{i}-1)} \cdot \mathrm{R} \cdot 10^{\left(\frac{\alpha \mathrm{L}}{5}+1\right)} \cdot \mathrm{P}_{\mathrm{In}}
$$

Where $\mathrm{R}$ is the Rayleigh scattering coefficient of the SMF within a pulse.

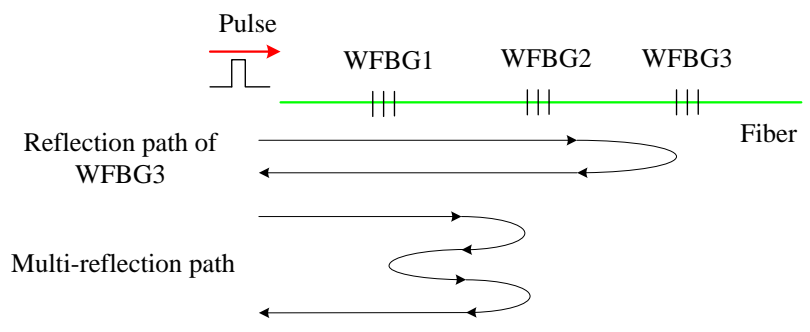

Fig. 2: Paths of WFBGs reflection

To distinguish the reflected signal from WFBGs and Rayleigh scattering, the power of the reflected light by WFBGs should be higher than the Rayleigh scattering light. Meanwhile, because of the limitation of the measuring ranges of PD and acquisition card, the light power difference between the reflected from the WFBGs and the Rayleigh scattering should not exceed the measuring ranges of PD and acquisition card, thus:

$$
1<\mathrm{r} / \mathrm{R}<\mathrm{P}_{\mathrm{M}}
$$

Where $\mathrm{P}_{\mathrm{M}}$ is the dynamic range of $\mathrm{PD}$ and acquisition card.

The ratio of the multi-reflected and Rayleigh scattering power can be obtained as:

$$
\operatorname{Pr}=10 \cdot \log \left(\frac{\mathrm{P}_{\text {OUT } 2}}{\mathrm{P}_{\text {OUTR }}}\right)=10 \cdot \log \left(\frac{(\mathrm{i}-1) \cdot(\mathrm{i}-2) \cdot \mathrm{r}^{3}}{2(1-\mathrm{r})^{2} \cdot \mathrm{R}}\right)
$$

The simulation from Eq.13 is shown in Fig.3. The ratio of multi-reflected and Rayleigh scattering power increases with the number of WFBGs. In order to distinguish the Rayleigh scattering signal from the multi-reflected lights and avoid crosstalk, the lights experience multi-reflection paths should far less than the Rayleigh scattering signal within one pulse. As shown in Fig.3, when the reflectivities of WFBGs are $5 \times$ $10^{-5}, 10^{-5}$, and $5 \times 10^{-6}$, the maximum numbers of WFBGs integrated in the proposed system are 401, 4472 and 12650, respectively.

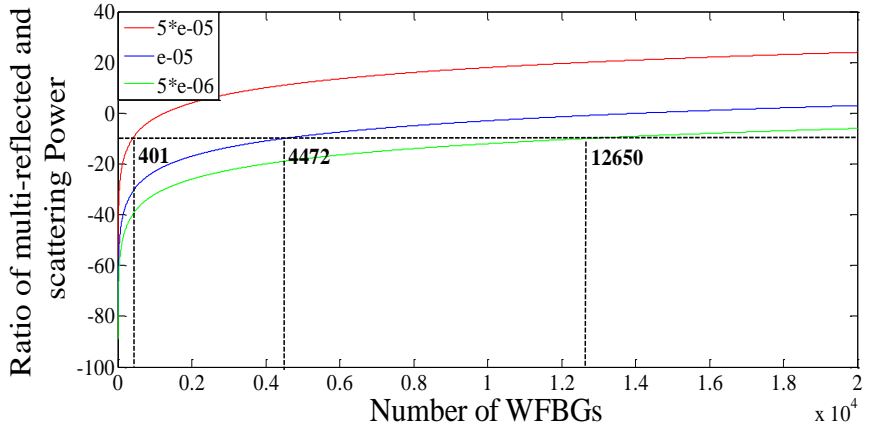

Fig. 3: The maximum number of WFBGs with different reflectivities.

\section{EXPERIMENTAL RESULTS}

\section{A. Experimental setup}

The LPG used in the sensing system has several broad transmission loss dips with steep rising and falling edges, which made it ideal to be used as a linear filter. The transmission spectrum of the LPG is plotted in Fig.4, showing two transmission loss peaks between $1500 \mathrm{~nm}$ and $1560 \mathrm{~nm}$. The linear response region on the spectrum edge around 1550 $\mathrm{nm}$ is marked in the inset in Fig.4. The loss amplitude is linear with wavelength from $1549 \mathrm{~nm}$ to $1551 \mathrm{~nm}$. When the light travels through the LPG, the output power would linearly change with the wavelength of the input light, which is a typical linear filter. As it is known, the LPG is very sensitive to the environmental condition [13], because the loss peaks are produced by the core mode coupling to the cladding modes which are much closer to the surrounding medium. In order to maintain the filter stability, the LPG was fixed on an aluminum plate during the experiment.

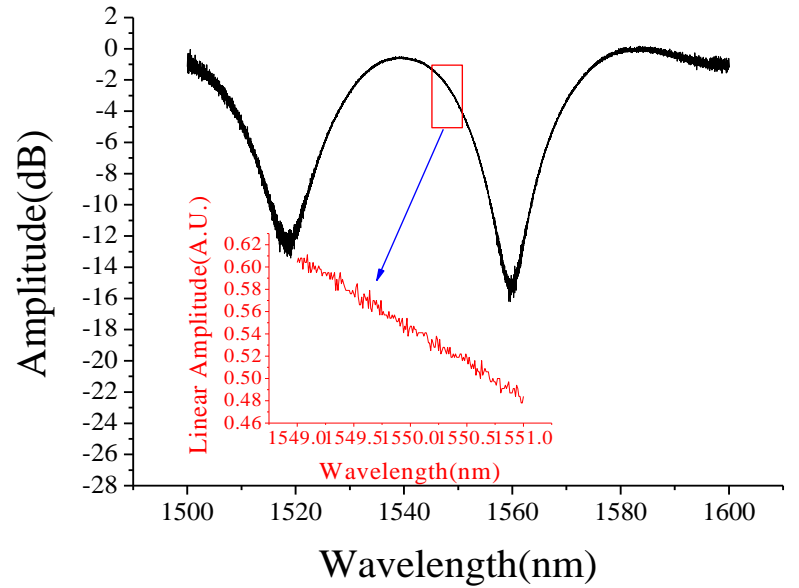

Fig.4: The transmission spectrum of the LPG. Inset: Zoomed spectrum between $1549.0 \mathrm{~nm}$ to $1551.0 \mathrm{~nm}$, showing linear response.

A schematic diagram of the experimental system used to verify the performance of the proposed system is shown in Fig.5. Light with $1.7 \mathrm{~nm}$ bandwidth generated by a broadband source and a chirped FBG is modulated into 100 ns pulse using an electro-optic modulator (EOM) and amplified by an EDFA. The amplified pulse with ASE filtered by a similar chirped FBG is injected into a $2.6 \mathrm{~km}$ sensing fiber with 3 WFBGs which were UV-inscribed at the end section of the sensing fiber with $50 \mathrm{~m}$ separation. The reflected light from WFBGs and back Rayleigh scattering light passed through LPG can be converted to electrical signal by a PD. Then the electrical signal is sampled by the acquisition card for further processing.

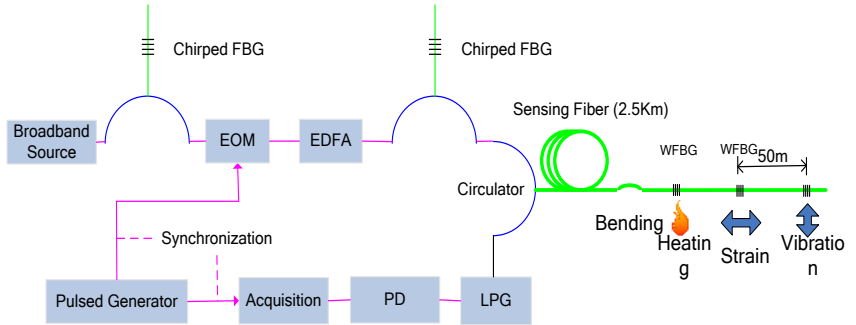

Fig.5: The experimental setup of the combined system based on OTDR and gratings. 
As the sensing range of WFBGs is limited by the bandwidth of the input light, a broader bandwidth is desirable, and the bandwidth of the input light source should not exceed the linear range of the LPG spectrum to maintain the wavelength-to-power conversion. . Meanwhile, the effective spectrum of the input light source should be as flat as possible to ensure the linearity and sensitivity of WFBGs sensing system. As shown in Fig.6, the bandwidth of the input light in our experiment is about $1.7 \mathrm{~nm}$ while the power fluctuation of the effective spectrum is about $0.8 \mathrm{~dB}$. As the power of the effective spectrum is almost linearly increasing with wavelength, assuming the slope of the power within the effective spectrum is $\mathrm{C}_{\mathrm{e}}$, Eq. 1 can be further described as:

$$
\mathrm{P}_{\text {OUT }}=\left(\mathrm{C}_{1} \lambda_{\mathrm{i}}+\mathrm{C}_{2}\right) \mathrm{P}_{\mathrm{In}}=\left(\mathrm{C}_{1} \lambda_{\mathrm{i}}+\mathrm{C}_{2}\right)\left(1+\mathrm{C}_{\mathrm{e}}\right) \mathrm{P}_{\mathrm{e}}
$$

Where $\mathrm{P}_{\mathrm{e}}$ is the fiducial power of the effective spectrum. As shown in Eq.14, the power of the output light is still linearly increasing with the wavelength. Thus, as the slope increases from $C_{1}$ to $C_{1} \cdot\left(1+C_{e}\right)$, the sensitivity of the sensing system can be improved due to this power fluctuation of the effective spectrum.

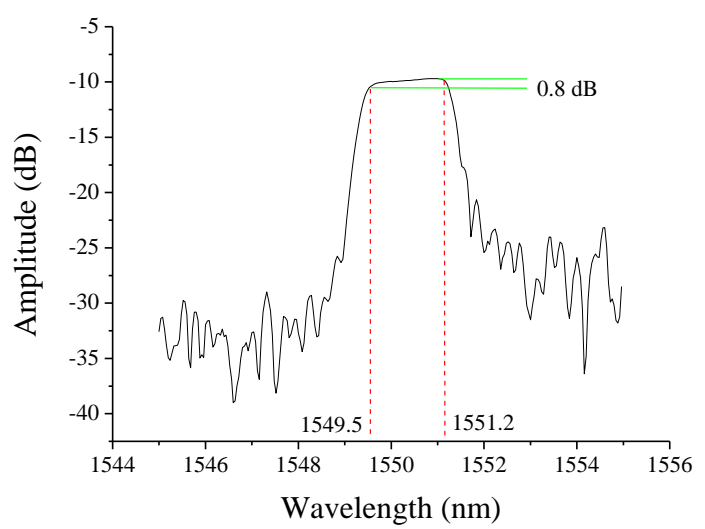

Fig.6: The spectrum of the input light generated by the broadband source and chirped FBG.

The length of each WFBG was fabricated as long as possible to obtain narrow spectrum, which can increase the accuracy of wavelength demodulation by the LPG. Then the power of the light passed through LPG can be easier calculated because the WFBG reflected light can be considered as single wavelength light. In our experiment, the length of WFBG is about $20 \mathrm{~mm}$, and the bandwidth of each WFBG spectrum is about $40 \mathrm{pm}$ as shown in Fig.7. The Bragg wavelengths of the 3 WFBGs are at $1550.35 \mathrm{~nm}, 1550.49 \mathrm{~nm}$ and $1550.68 \mathrm{~nm}$ respectively. As mentioned above, the wavelengths of WFBGs can be arbitrary as long as it is within the bandwidth of the input laser and linear spectral region of the LPG.

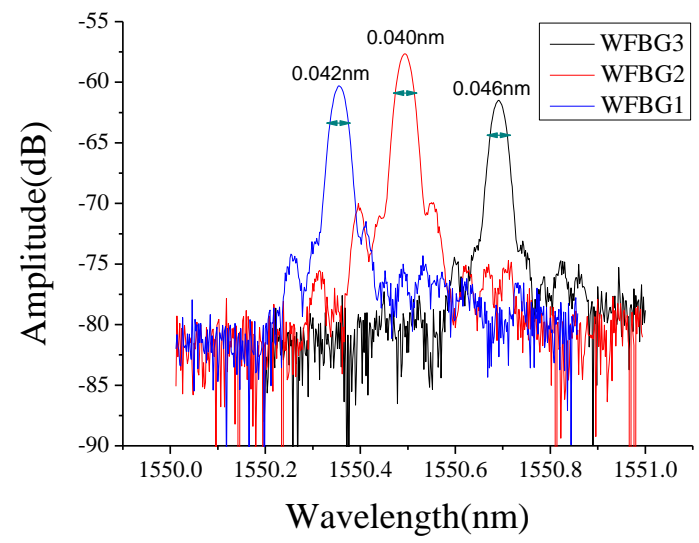

Fig.7: The reflected spectra of the 3 WFBGs.

Using the OTDR technology with 100 ns pulse (the spatial resolution is $10 \mathrm{~m}$ ), the distribution of 3 WFBGs is obtained as shown in Fig.8, where the position and reflectivity information of WFBGs can be obtained. The strong signal at the beginning of the OTDR curve is the Fresnel reflection. 3 WFBGs are located at $2555 \mathrm{~m}, 2605 \mathrm{~m}$ and $2655 \mathrm{~m}$, respectively. The amplitude of WFBG reflected light is about $177 \mathrm{mV}$ while the amplitude of Rayleigh scattering light around $2500 \mathrm{~m}$ is about $40 \mathrm{mV}$. The WFBG reflected light and Rayleigh scattering light are not only generated by the pulse but also by the DC light of the optical pulse and ASE noise, as the signal out of sensing fiber is about $29 \mathrm{mV}$ as shown in Fig.8. Therefore, the signal generated by DC light should be subtracted to calculate the reflecting coefficient of WFBGs. Thus three reflected lights are about $\quad \frac{170 \mathrm{mV}-29 \mathrm{mV}}{40 \mathrm{mV}-29 \mathrm{mV}} \approx 12.8, \frac{177 \mathrm{mV}-29 \mathrm{mV}}{40 \mathrm{mV}-29 \mathrm{mV}} \approx$ 13.5 and $\frac{75 \mathrm{mV}-29 \mathrm{mV}}{40 \mathrm{mV}-29 \mathrm{mV}} \approx 4.2$ times higher than Rayleigh scattering light. Because the Rayleigh scattering coefficient is about $10^{-6}$ for $10-\mathrm{m}$ fiber, the reflectivities of the three WFBGs were estimated to be about $1.28 \times 10^{-5}, 1.35 \times$ $10^{-5}$, and $0.42 \times 10^{-5}$. As analyzed in section III, about 4000 WFBGs can be integrated in our proposed system. As the interval of WFBGs is about $50 \mathrm{~m}, 200 \mathrm{~km}$ sensing fiber can be monitored theoretically. Actually, due to the limitation of dynamic range of PD and the signal to noise ratio (SNR) of the system, the sensing distance would be less than $200 \mathrm{~km}$.

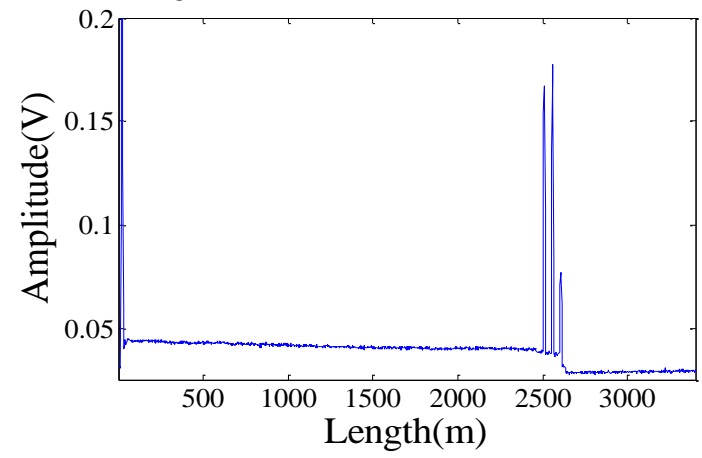

Fig.8: The time domain signal of the sensing fiber with 3WFBGs.

To evaluate the potential sensing applications of this system, we have investigated the temperature, strain and vibration sensing properties around WFBGs and distributed bending sensing property along the whole sensing fiber. 


\section{B. Temperature and strain sensing on key-positions}

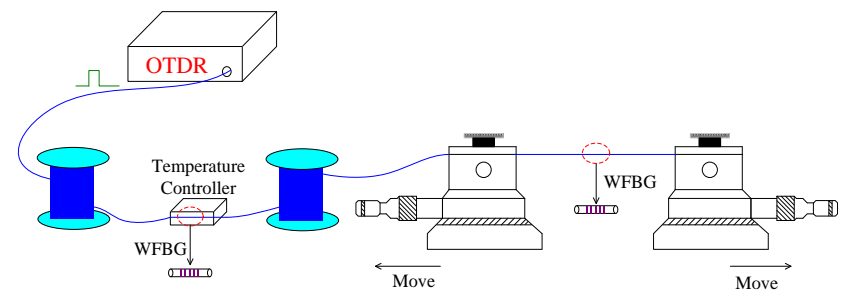

Fig.9: Experiment setup for strain and temperature sensing characterization.

As shown in Fig.5 and Fig.9, the first WFBG is subjected to temperature sensing, while the strain is applied on the second WFBG by two micro-adjustable stages to verify key-points sensing in the experiment. The fiber is fixed on the stages with both sides while moving one of the stages to stretch the WFBG as shown in Fig.9.

When the temperature on the first WFBG and strain on the second WFBG are changed, the power of the light will also change accordingly after passing through the LPG. The temperature sensing performance of the first WFBG and the strain sensing performance of the second WFBG are shown in Fig.10 and Fig.11, respectively. From the figures, we can estimate the temperature and strain sensitivities are about $5.9 \times$ $10^{-3} /{ }^{\circ} \mathrm{C}$ and $4.2 \times 10^{-4} / \mu \varepsilon$ respectively. As analyzed above, the sensitivity depends on two parameters $\mathrm{K}_{\mathrm{f}}$ and $\mathrm{C}_{1}$. The first one depends on the fiber which is fixed when the fiber is chosen. The second one depends on the gradient of the linear filter which can be improved by fabricating LPG with higher stop band attenuation rate.

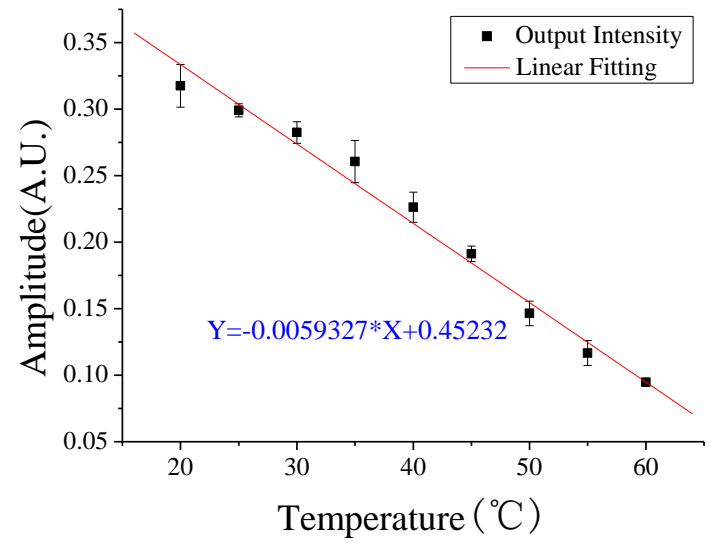

Fig.10: The temperature sensing of the first WFBG.

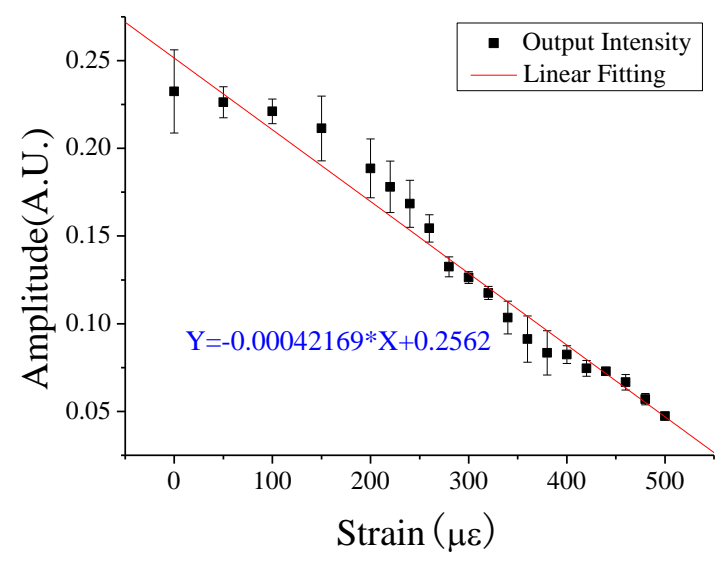

Fig.11: The strain sensing of the second WFBG.
The resolution of the sensing system mainly depends on the resolution of the $\mathrm{A} / \mathrm{D}$ converter which is limited by the bit of the data acquisition card. A 10 bits data acquisition card was used in our experiment. Thus the best achievable resolution values for temperature and strain sensing using our system are about $\frac{1 / 2^{10}}{5.9 \times 10^{-3} /{ }^{\circ} \mathrm{C}} \approx 0.17{ }^{\circ} \mathrm{C}$ and $\frac{1 / 2^{10}}{4.2 \times 10^{-4} / \mu \varepsilon} \approx 2.32 \mu \varepsilon$ respectively.

According to the linear conversion function, the amplitude of the output signal changes by about $25 \mathrm{mV}$ when the wavelength of WFBG changes by $0.44 \mathrm{~nm}$ as shown in Fig.10. Therefore the largest amplitude change is about $97 \mathrm{mV}$ when the wavelength of the WFBG shifts by $1.7 \mathrm{~nm}$ which is the bandwidth of the laser. Then the temperature and strain sensing ranges are about $153{ }^{\circ} \mathrm{C}$ and $1938 \mu \varepsilon$, which can be improved by choosing laser with wider bandwidth. There is a mutual restraint between the stop band attenuation rate and the filter range as the dynamic range is fixed when the $\mathrm{PD}$ and data acquisition card are chosen. However, the sensitivity and the sensing range can be further improved by designing the linear property of LPG.

The linearity of the curves $\left(\mathrm{R}^{2}\right)$ in Fig.10 and Fig.11 are about $99.12 \%$ and $98.36 \%$, respectively. In addition, the mean deviation of the temperature sensing is about $2.57 \%$ while that of the strain sensing is about $3.87 \%$. These deviation coefficients could be caused by three facts. Firstly, there may exist residual spectral instability of the LPG induced by the surrounding environmental change. Secondly, the strain applied on the fiber may not be accurate enough due to the fiber sliding effect. These two eefects could be improved by designing a better LPG housing and WFBG loading. Last but not least, spectral flatness and linearity of the spectrum of the light source and the noise induced by APD may also induce extra errors during the measurement. This deviation can be reduced by using a light source with more flat spectrum.

\section{Quasi-distributed vibrations sensing}

In many practical applications, the static temperature and strain monitoring is not enough. Dynamic event such as vibration detection is also important. In our experiment, singleand multiple-vibration monitoring have been demonstrated.

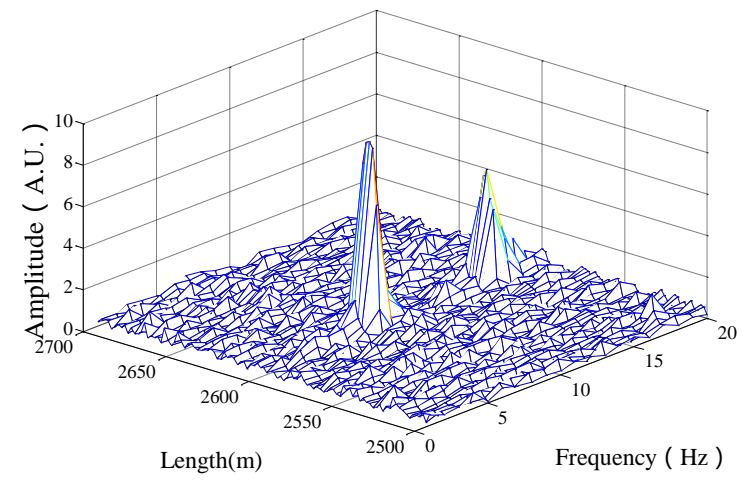

Fig.12: Detected vibration with $8 \mathrm{~Hz}$ at $2605 \mathrm{~m}$.

To demonstrate the vibration sensing principle and evaluate the performance of the proposed system, vibrations with different frequencies were loaded on WFBGs respectively. The 
signal for each position can be transferred into frequency domain by FFT to obtain the vibration frequency spectra along the sensing fiber. In the experiment, $8 \mathrm{~Hz}$ frequency vibration was applied on the second WFBG. As shown in Fig.12, the retrieved frequency spectra of each point along the sensing fiber clearly show that the spectrum at the second WFBG position has the frequency component of $8 \mathrm{~Hz}$ which is equal to the frequency of the vibration we have applied. Then the location and frequency information of the vibration can be obtained by tracking the spectra along the whole sensing fiber. It should be noticed that there is also signal at $16 \mathrm{~Hz}$ which is the double frequency of $8 \mathrm{~Hz}$ at $2605 \mathrm{~m}$. It is caused by the shake of fiber while loading the vibration in the experiment. The double frequency signal can be avoided by improving the way of loading the vibration and laying the sensing fiber.

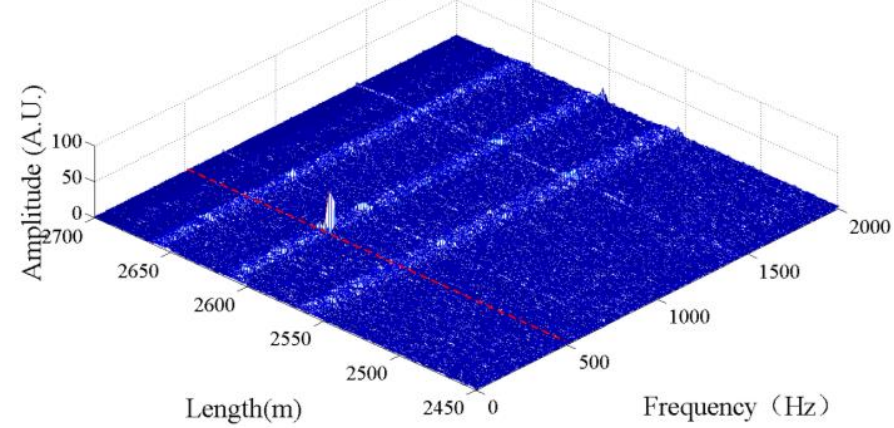

Fig.13: Detected vibrations with $500 \mathrm{~Hz}$ at $2605 \mathrm{~m}$.

Because the length of the sensing fiber in our experiment is about $2.7 \mathrm{~km}$, the theoretical highest frequency of the vibration that can be detected is about $2.25 \mathrm{kHz}$ with 8 times average according to Eq.8. However, the highest vibration frequency can be loaded on the WFBG is $500 \mathrm{~Hz}$ by using a woofer in our lab, which is enough to show the sensing performance of the proposed system for high frequency vibration. In the experiment, the $500 \mathrm{~Hz}$ frequency vibration was imposed to the second WFBG, and results in Fig.13 have clearly shown the system has detected $500 \mathrm{~Hz}$ vibration at $2605 \mathrm{~m}$ position. In Fig.13, besides the $500 \mathrm{~Hz}$ frequency component at $2605 \mathrm{~m}$, there are 700 and $1500 \mathrm{~Hz}$ frequency components along the whole sensing fiber, which may be induced by the power fluctuation of the light source or the spectrum shift of LPG. As these frequency components also appear at the positions of non-WFBG, it can be directly ignored in detecting vibrations along WFBGs. In addition, as the power of the reflected light from 3 WFBGs is much higher than the Rayleigh scattering, the noise at these 3 WFBGs positions is also relatively higher, which can be reduced by more averages.

Multi-vibrations monitoring has also been achieved in our proposed system. When we applied another $11 \mathrm{~Hz}$ frequency vibration on the second $(2605 \mathrm{~m})$ and third WFBG $(2655 \mathrm{~m})$ simultaneously, the retrieved spectra along the fiber showed two vibrations detected at $2605 \mathrm{~m}$ and $2655 \mathrm{~m}$ (see in Fig.15). As the spectra at the key points are independent, more vibrations can be detected simultaneously.

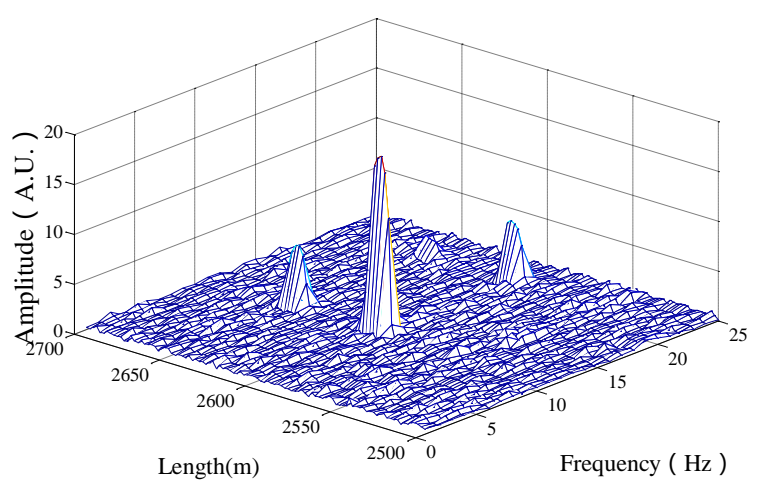

Fig.14: Vibrations with $11 \mathrm{~Hz}$ at $2605 \mathrm{~m}$ and $2655 \mathrm{~m}$ simultaneously.

\section{D.Distributed bending sensing}

In our proposed system, distributed bending sensing has also been demonstrated. When the fiber is disturbed by bending, the power of the back Rayleigh scattering after the bend would be reduced. Therefore, it is easily to distinguish the information of bending by detecting the power change of Rayleigh back scattering along the fiber.

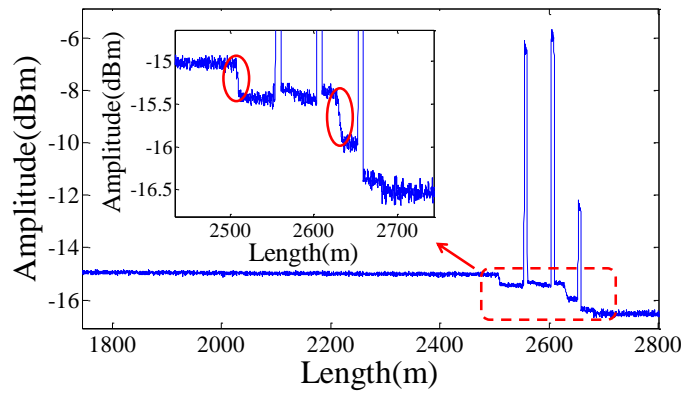

Fig.15: The distributed time domain signal: 2 bends at $2500 \mathrm{~m}$ and $2630 \mathrm{~m}$ simultaneously.

In the experiment, the sensing fiber was bent at $2500 \mathrm{~m}$ and $2630 \mathrm{~m}$ simultaneously. The Rayleigh scattering along the fiber is shown in Fig.15. The power of the Rayleigh scattering is reduced at the two positions as shown in the two red circle areas in Fig.15. And the corresponding losses are about $-0.4 \mathrm{~dB}$ and $-0.6 \mathrm{~dB}$ respectively. Meanwhile, the position information of bends could be located by analyzing the point of the power change of the Rayleigh scattering. Therefore distributed bend sensing is achieveable by the proposed system. In future work, ultra weak-FBGs could be fabricated over entire sensing fiber to achieve long distance and more key-positions sensing to greatly enhance the sensing network capacity.

\section{V.CONCLUSION}

We have demonstrated a distributed fiber sensing system with capability of monitoring key-positions along the fiber integrated with WFBGs, LPG and OTDR technologies. In the sensing system, the Rayleigh scattering signals were detected for distributed bend detection while the WFBGs were used for temperature, strain and vibration sensing on key-positions. The location information of WFBGs and bends were obtained by the OTDR. Without demodulating the wavelength shift, the temperature and strain information were obtained simply by measuring the power of the reflected light after passing an LPG based linear filter. In the system, we used 3 WFBGs to verify 
the capability of system for both distributed and key-position sensing. However, the potential largest capacity of the sensing network has already been theoretically analyzed, which is possible to integrate more than 4400 WFBGs in the system. The experimental results show the strain and temperature sensitivities of $4.2 \times 10^{-4} / \mu \varepsilon$ and $5.9 \times 10^{-3} /{ }^{\circ} \mathrm{C}$ respectively. Detection of multiple vibrations with up to $500 \mathrm{~Hz}$ frequency band is also achieved at WFBG positions. Finally, we demonstrated distributed bend sensing using the proposed system.

\section{REFERENCES}

[1] Hill, K.O. and G. Meltz, "Fiber Bragg grating technology fundamentals and overview," J. Lightw. Technol., vol.15, no.8, pp. 1263-1276, Aug. 1997.

[2] Bao, X. and L. Chen, "Recent progress in distributed fiber optic sensors," Sens., vol.12, no.7, pp. 8601-8639, Jun. 2012.

[3] Kurashima T., Tateda M., Shimizu K., Horiguchi T. and Koyamada Y., "A high performance OTDR for measuring distributed strain and optical loss," ECOC'96, pp.215-218, 1996.

[4] Edvard C. and Denis D., "In-line short cavity Fabry-Perot strain sensor for quasi distributed measurement utilizing standard OTDR," Opt. Express, vol.15, no.14, pp.8719-8730, Jul. 2007.

[5] J. Kou, S. Qiu, F. Xu and Y. Lu, "Demonstration of a compact temperature sensor based on first order Bragg grating in a tapered fiber probe," Opt. Express, vol.19, no.19, pp.18452-18457, Sep. 2011.
[6] Wu Y., Stefani A. and Bang, O., "Tunable polymer fiber Bragg grating inscription: Fabrication of dual-FBG temperature compensated polymer optical fiber strain sensors," IEEE Photon. Technol. Lett., vol.24, no.5, pp.401-403, Mar. 2012.

[7] Gao, H., Li H., Liu B., Zhang H., Luo J., Cao Y., Yuan Sh., Zhang W., Kai G. and Dong X., "A novel fiber Bragg grating sensors multiplexing technique," Opt. Comm., vol.251, no.4, pp. 361-366, Jul. 2005.

[8] Y. Wang, J. Gong, B. Wang, W. Bi and A. Wang, "A quasi-distributed sensing network with time- division- multiplexed fiber Bragg gratings," IEEE Photon. Technol. Lett., vol.23, no.2, pp.70-72, Jan. 2011.

[9] Zhang M., Sun Q., Wang Z., Li X., Liu H. and Liu D., "A large capacity sensing network with identical weak fiber Bragg gratings multiplexing," Opt. Comm., vol.285, no.13, pp. 3082-3087, 2012.

[10] Hu C., Wen H., and Bai W., "A novel interrogation system for large scale sensing network with identical ultra-weak fiber Bragg gratings," J. Lightw. Technol., vol.32, no.7, pp. 1406-1411, Apr. 2014.

[11] Li X., Sun Q., Liu D., Liang R., Zhang J., Wo J., Shun P., Liu D., "Simultaneous wavelength and frequency encoded microstructure based quasi-distributed temperature sensor," Opt. Express, vol.20, no.11, pp.12076-12084, May, 2012.

[12] S. Tanaka, O. Tsukida, M. Takeuchi, S. Tekuramori, R. Uchimura, A. Wada and N. Takahashi, "Highly sensitive operation of intensity based fiber optic vibration sensor using cascaded long period fiber grating," Proc. SPIE 9157 OFS, vol.9157, 2014.

[13] R. W. Fallon, L. Zhang, L.A. Everal, J.A.R. Williams and I. Bennion, "All fibre optical sensing system: Bragg grating sensor interrogated by a long period grating," Meas. Sci. Technol., vol.9, no.12, pp.1969-1973, Dec. 1998. 